\title{
Spatial and temporal factors in the perception of ethanol irritation on the tongue
}

\author{
BARRY G. GREEN \\ Monell Chemical Senses Center, Philadelphia, Pennsylvania
}

\begin{abstract}
The sensitivity of the tongue to the irritation produced by ethanol was measured in five experiments. It was discovered in the first two experiments that both latency to the onset of irritation and perceived intensity of irritation varied with the locus of lingual stimulation. The tonguetip and the side of the tongue were found to be more sensitive (that is, they tended to produce higher magnitude estimates and shorter response latencies) than sites toward the middle of the tongue, with the tonguetip being the most sensitive area tested. However, even on the tonguetip the latency to onset of irritation (approximately 2-6 sec) was much longer than typical values for taste sensation. The third experiment demonstrated that increasing the size of the ethanol stimulus resulted in shorter response latencies and more intense irritation. The latter result indicates that the sensory system responsible for ethanol irritation is capable of significant (but incomplete) spatial summation. The final two experiments showed that significant summation also occurs when stimulus area is increased by adding a second stimulus at another lingual locus. Possible explanations for the regional differences in sensitivity and for the relatively long onset latencies of chemical irritation are discussed.
\end{abstract}

Although ethanol (ethyl alcohol) is among the most common oral chemical irritants, little is known about the nature of the sensations it produces. The absence of knowledge about ethanol's sensory effects is surprising given the ubiquity of alcohol consumption, but unsurprising given the state of knowledge about oral chemical irritation in general. Only recently have the sensory and perceptual characteristics of chemical irritation in the mouth begun to be explored systematically, and ethanol has not been among the compounds studied. Researchers have usually focused instead on two irritants that appear in peppers: capsaicin (e.g., Green, 1986; Lawless, 1984; Rozin, Ebert, \& Schull, 1982; Stevens \& Lawless, 1986) and piperine (e.g., Lawless, 1984).

Interest in the perception of ethanol has generally centered on its taste, not its irritation (e.g., Martin \& Pangborn, 1970; Smith, 1972; Wilson, O'Brien, \& MacAirt, 1973). It appears that the only data on the irritation produced by ethanol have come from an early, unsystematic survey of the local sensitivity of oral areas to ethanol (Parker \& Stabler, 1913) and a more recent series of measurements on the threshold for the "burning taste" during whole-mouth stimulation (Wilson et al., 1973). The study by Parker and Stabler provided little quantitative information about ethanol irritation, and the

The author thanks Mary Ellen Bailey for conducting the experiments in this study and for assisting in data reduction and analysis. The research was supported in part by Grants NS20577 and NS20616 from the National Institutes of Health. Portions of these data were reported at the International Symposium on Olfaction and Taste held in Snowmass, CO, July 1986, and at the ninth meeting of the Association for Chemoreception Sciences, Sarastoa, FL, May 1987. Requests for reprints should be sent to the author at the Monell Chemical Senses Center, 3500 Market Street, Philadelphia, PA 19104. sensitivity of the tongue was not measured because the researchers wanted to avoid stimulating "gustatory organs" while assessing chemical irritation. The study by Wilson et al. provided information about the sensitivity of the whole mouth to irritation, but it was not intended to reveal anything about the sensitivity of specific oral structures.

The primary objective of the present study was to assess the effects of stimulus location and size on the tongue's sensitivity to ethanol irritation. Sensitivity was defined in terms of two perceptual variables: latency to sensation onset (as inferred from response time) and perceived intensity of irritation. Experiments 1 and 2 were surveys of the sensitivity of the tongue in the longitudinal (rostrocaudal) and transverse planes. Experiments 3-5 investigated the effect on perceived intensity of increasing the area of stimulation. One objective of the latter experiments was to determine whether the sensory systems that are sensitive to ethanol exhibit spatial summation, and hence whether local differences in sensitivity that had been found in the first two experiments could be attributed in part to local differences in innervation density. A second objective was to reveal whether, with respect to spatial summation, chemical irritation is more closely allied to the senses of warmth and cold (which are characterized by large and consistent amounts of summation) or to the sensitivity to heat pain (which appears to show less robust summation). In Experiment 3 the size of a single stimulus was varied, and in Experiments 4 and 5 stimulus area was doubled by the addition of a second stimulus at a contralateral lingual site. Both methods of increasing stimulus area resulted in partial, but statistically significant, spatial summation. 


\section{EXPERIMENT 1}

The purpose of this experiment was to compare the sensitivity to ethanol on the anterior and medial areas of the dorsal surface of the tongue. Evidence from several sources had indicated that the anterior dorsal surface should be more sensitive to ethanol than the medial dorsal surface. Psychophysical data had shown that the perception of both warmth and heat pain was stronger near the tonguetip than it was a few centimeters posterior to the tip (Green, 1984, 1985). This finding is relevant to the present study because the sensation produced by ethanol is typically referred to as "warmth" or "burn," and because there is evidence from percutaneous recordings in humans that sensitivity to at least some chemical irritants is mediated by afferent fibers that respond to high skin temperatures (Konietzny \& Hensel, 1983; Van Hees \& Gybels, 1981). In addition, sensitivity to capsaicin had been reported to be present on the anterior but absent on the posterior region of the tongue, a result that coincided with the spatial differences in the density of substance-P immunoreactive neurons in lingual epithelial tissue in humans (Duner-Engstrom, Fredholm, Larsson, Lundberg, \& Saria, 1986). If the sensory responses to capsaicin and ethanol are mediated at least in part by the same population(s) of afferent fibers, then the sensitivity to the two substances should be distributed similarly over the surface of the tongue.

\section{Method}

Subjects. Fifteen young adults (11 females and 4 males; average age, 23 years) were paid to participate.

Apparatus and Procedure. The stimuli were $0.38-\mathrm{cm}^{2}$ disks of filter paper (Whatman No. 1) saturated with one of six concentrations of ethyl alcohol: $35 \%, 45 \%, 55 \%, 65 \%, 75 \%$, or $85 \%$ (by volume). The solutions, which contained deionized water as the diluent, were stored in glass vials with screw tops. In the testing sessions, the screw tops were replaced with small pieces of Plexiglas both to facilitate access to the solutions and to shorten the time the solutions were exposed to air during each trial. The experimenter was able to lift the plastic cover with one hand, dip a paper disk (held by forceps) into the ethanol solution with the other hand, then replace the cover and quickly apply the paper to the subject's tongue. The subject sat a few feet away and was shielded from the preparation area by a cardboard divider. The entire process of stimulus application (which included drawing the filter paper across the lip of the vial to remove excess solution) took 3 or $4 \mathrm{sec}$.

The stimuli were placed on two sites along the midline of the dorsal surface of the tongue: at the tonguetip and $3 \mathrm{~cm}$ posterior to the tip. The latter location was marked by a drop of red food color placed adjacent to the midline. (This method enabled repeated stimulation within a few millimeters of the target location. More accurate placement was not possible because the food color tended to smear during the session.) The subject was told to retract the tongue and close the mouth (resting the tongue on the floor of the mouth) as soon as the filter paper was placed on the tongue. The mouth was kept closed to reduce the rate at which the ethanol evaporated and to maintain the temperature of the tongue near normal oral temperature. Maintenance of normal temperatures was important because informal observations had indicated that, like the irritation produced by capsaicin (Green, 1986), the irritation produced by ethanol could be significantly attenuated by cooling.
Two sensory measures were collected: latency to sensation onset and perceived magnitude. Estimates of latency to onset of irritation were obtained by having the subjects press a key twice, once when they felt the tactile sensation produced by application of the filter paper to the tongue and again when they felt the sensation of irritation or burn begin. The time between keypresses (calculated by a computer) was taken as the measure of latency. The subjects were instructed to avoid responding to the presence of sensations other than irritation (e.g., coolness or taste). It had been discovered in pilot work that some individuals were unable to detect irritation on the medial region even if the filter paper was left on the tongue for $1 \mathrm{~min}$ or more. This necessitated the imposition of a 60 -sec limit on the "observation interval" during which the subject could respond to the presence of irritation. Failure to respond within $60 \mathrm{sec}$ resulted in the recording of an infinite latency and a magnitude estimate of zero.

When irritation was detected, a magnitude estimate of the maximum intensity of irritation perceived during the trial was reported verbally immediately after the filter paper was removed from the tongue. The paper was always removed approximately $10 \mathrm{sec}$ after the subject indicated that irritation had been felt. This timing was achieved by having the computer sound a tone $9.5 \mathrm{sec}$ after the subject's second keypress. Upon hearing the tone the subject opened his/her mouth and the experimenter plucked the filter paper from the tongue (using forceps). The signal at $9.5 \mathrm{sec}$ guaranteed stimulation would last at least $10 \mathrm{sec}$ (it took the experimenter more than $0.5 \mathrm{sec}$ to remove the filter paper), but not significantly longer. Hence, regardless of the latency to sensation onset, irritation was perceived for the same duration for every stimulus.

The subjects rinsed between trials with deionized water that had been warmed to $38^{\circ} \mathrm{C}$ in a constant-temperature bath. This practice helped wash the remaining ethanol from the tongue and maintained the temperature of the mouth near $37^{\circ} \mathrm{C}$. Trials were separated by $60 \mathrm{sec}$ (timed from the beginning of rinsing) to allow lingering sensations of irritation to subside. To protect further against possible sequential effects, the location of stimulation was alternated from trial to trial; stimulation at a given locus was therefore separated by a minimum of $2 \mathrm{~min}$. Stimulus concentration was varied pseudorandomly over trials, with the constraint that no concentration be applied a second time to a given site until every other concentration had been presented to that site at least once (all concentrations were eventually presented twice to each location).

\section{Results and Discussion}

The median response latencies for the two areas of the tongue are shown in Figure 1. Medians were calculated rather than means because response latencies varied substantially across subjects and because 3 subjects failed to feel irritation at the medial site within $60 \mathrm{sec}$ when some of the lower concentrations were applied.

The data show that the irritation produced by ethanol was perceived sooner on the tip of the tongue than $3 \mathrm{~cm}$ posterior to the tip [Friedman test, $\chi^{2}(1)=12.0$, $p<.001]$. The median response time for the lowest concentration tested (35\%) was $5.9 \mathrm{sec}$ on the tip of the tongue. In contrast, the median response time on the medial area was $21.6 \mathrm{sec}$. The latencies to sensation onset shortened on both areas as concentration increased, falling to a median value for the $85 \%$ solution of $1.5 \mathrm{sec}$ on the tonguetip and $9.5 \mathrm{sec}$ on the medial region. At the highest concentration tested, the two loci therefore differed with respect to latency to sensation onset by a factor of more than 6 to 1 . 


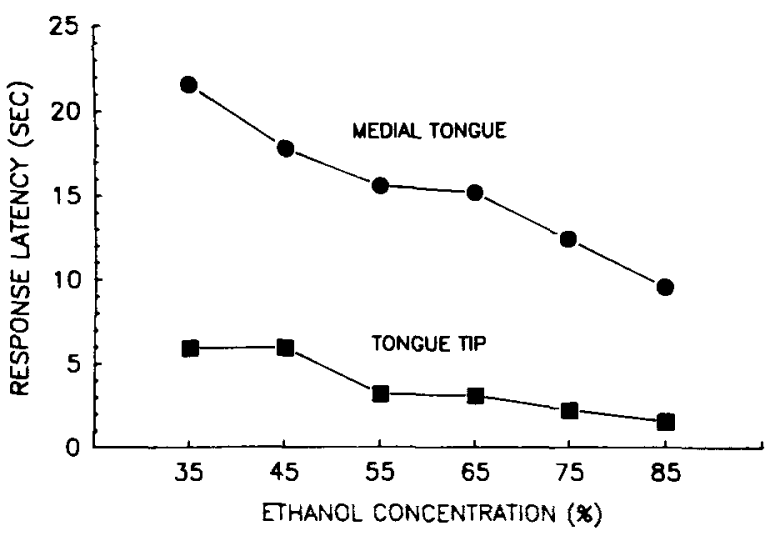

Figure 1. Latency to detection of irritation as a function of ethanol concentration for two areas of the tongue: the tip and $3 \mathrm{~cm}$ posterior to the tip. The data are medians.

Figure 2 displays the results from the magnitude estimation task. The data have been normalized by calculating the mean magnitude estimate for each subject in the tonguetip condition, dividing that mean into 10 , and multiplying each of the raw scores in both conditions by the resulting factor. The data reported are arithmetic means.

It can be seen in Figure 2 that the sensitivity to the irritation produced by ethanol exhibits a steep rostrocaudal gradient. At every concentration, irritation was stronger on the tonguetip than it was $3 \mathrm{~cm}$ posterior to the tip [Friedman test, $\chi^{2}(1)=15.0, p<.001$ ]. The ratio of perceived intensities at the two sites ranged from a minimum of 2.6 to 1 for the $85 \%$ stimulus to as much as 5.2 to 1 for the $65 \%$ stimulus.

It also appears that the relationship between ethanol concentration and perceived irritation is not a simple one. The data have been plotted on linear coordinates in Figure 2 because, to a first approximation, the data from both loci are reasonably well fit by linear functions (solid lines). However, the function relating concentration to

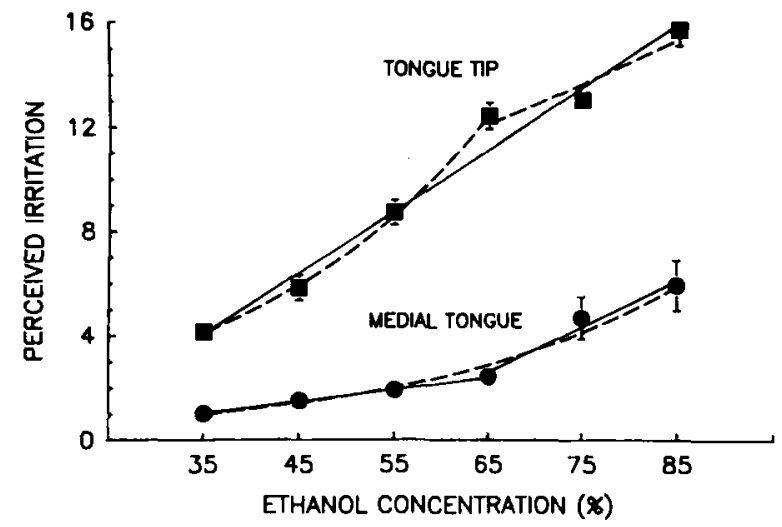

Figure 2. Mean perceived intensity of irritation as a function of ethanol concentration for sites on the tonguetip and dorsomedial tongue ( $3 \mathrm{~cm}$ posterior to the tip). Vertical bars indicate standard errors of the means. Solid lines = best-fitting linear functions; dashed lines $=$ best-fitting exponential functions. sensation on the medial locus has two distinct limbs, with a breakpoint at $65 \%$. The fit of the linear function on the lower limb is virtually perfect (Pearson $r=.999)$; the fit on the upper limb is somewhat less precise $(r=.986)$ and the variability around the individual data points is noticeably greater than at the lower concentrations. The intensity data for the medial locus can also be reasonably well described as a single exponential function $(r=.991)$. Exactly the opposite situation occurs for the data from the tonguetip. Although reasonably well fit by a single linear function $(r=.988)$, the data are fit better by two exponential functions (dotted lines in Figure 2). The exponential qualifies as a superior descriptor of the data over the lower concentrations because of the very high correlation associated with it $(r=.999)$, and because it accounts for what otherwise appears to be an aberrant datum at $65 \%$. That datum deviates noticeably from the linear function, and the small standard error of the mean associated with it implies that the deviation is reliable. The upper end of the function can also be fitted reasonably well with an exponential function $(r=.944)$, but less importance can be attached to this because the upper portion of the curve consists of only three data points. Of greater significance than the precise shape of the upper function is its mere presence; if the psychophysical functions for both lingual loci have two limbs, it may mean that more than one population of afferent fibers mediates the perception of ethanol irritation on the tongue.

\section{EXPERIMENT 2}

The second experiment explored the local sensitivity of the tongue in the transverse direction. Informal testing had revealed that the edge of the tongue was more sensitive to ethanol than was the middle of the tongue, suggesting that gradients of sensitivity existed in the transverse orientation as well as in the longitudinal orientation.

\section{Method}

Subjects. Fifteen young adults $(7$ males and 8 females; average age, 24.6 years) were paid to participate.

Apparatus and Procedure. As in the preceding experiment, the stimuli were pieces of filter paper on which a range of concentrations of ethanol were presented to the tongue. In this experiment, however, the stimuli were $0.5 \times 1.0 \mathrm{~cm}$ rectangles cut from larger pieces of filter paper (Whatman No. 1). This size and shape were used to facilitate comparison with another experiment (Experiment 3 ), in which stimulus size was varied by changing the size of rectangular pieces of filter paper. The concentrations used in the present experiment were the four highest ones tested in the first experiment: $55 \%, 65 \%, 75 \%$, and $85 \%$ (by volume).

The lingual loci tested were (1) the midline, (2) halfway between the midline and the edge (midlateral), and (3) the dorsal aspect of the edge (lateral). The sites were approximately $3 \mathrm{~cm}$ posterior to the tonguetip (again marked with food color), and the two lateral sites were to the right of the midline. The subjects received the stimuli in the manner of Experiment 1, and again pressed a key when they first felt a sensation of irritation at the test site. Also as before, estimates of perceived irritation were collected approximately $10 \mathrm{sec}$ after the onset of irritation, and subjects rinsed with deionized water $\left(38^{\circ} \mathrm{C}\right)$ during the 1 -min intertrial interval. 


\section{Results}

Median response latencies for the three areas tested are shown in Figure 3. The data indicate that sensations of irritation arose more rapidly near the edge of the tongue than on the midline of the tongue [Friedman test, $\chi^{2}(2)$ $=22.5, p<.0001]$. The intermediate locus (halfway between the midline and lateral sites) produced intermediate latencies. On average, the latencies on the medial locus and the most lateral locus differed by a ratio of approximately 2 to 1 . It may be recalled that the latencies obtained in Experiment 1 in the longitudinal direction differed by a ratio of as much as 6 . Because the same midline locus was stimulated in both experiments, it can be inferred that the greater differences in latencies along the tongue than across the tongue was primarily attributable to the superior sensitivity of the tonguetip compared with the edge of the tongue. The median latency to sensation onset for the $55 \%$ solution near the edge of the tongue was $7.8 \mathrm{sec}$; for the tip of the tongue it was $3.1 \mathrm{sec}$. On the midline, the same concentration produced more similar median latencies in both experiments: 15.5 and $13.5 \mathrm{sec}$.

The results for perceived intensity (Figure 4) do not show the same systematic differences across loci that were found for response latency. Although the mean perceived intensities (calculated using the normalization procedure of Experiment 1) were greater near the edge of the tongue than at either of the more medial sites, the overall effect of location failed to reach statistical significance when assessed either by a univariate repeated measures analysis of variance (ANOVA) $[F(2,28)=5.24$, $p=.012]$ or by the nonparametric Friedman test $\left[\chi^{2}(2)\right.$ $=16.13, p=.384]$. (The Friedman test was used in addition to the ANOVA because it had been applied to the data for response latency, which were not amenable to parametric analysis.) However, it is obvious from Figure 4 that in addition to producing larger mean magnitude estimates, the lateral location also yielded much larger standard errors of the means than did the other two

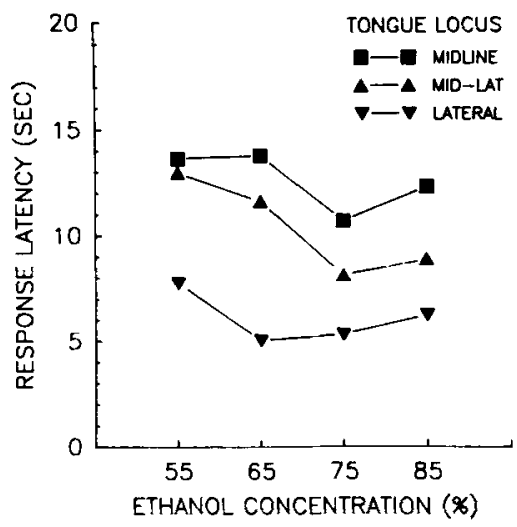

Figure 3. Latency to onset of irritation for three sites located $3 \mathrm{~cm}$ posterior to the tonguetip on the midline, halfway between the midline and the edge (midlateral), and on the dorsal aspect of the edge (lateral).

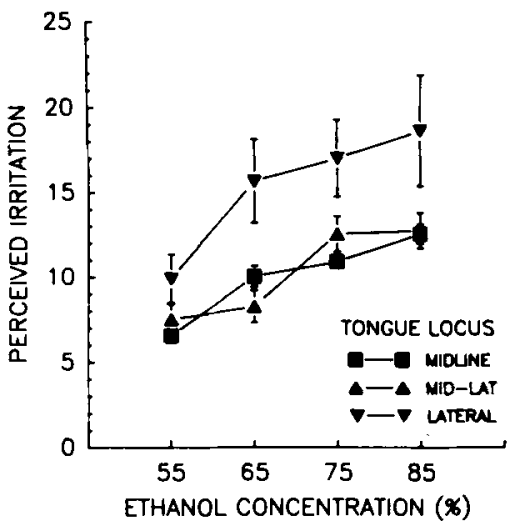

Figure 4. Mean perceived intensity of irritation as a function of ethand concentration for the three transverse sites of Figure 3. Vertical bars indicate standard errors of the means.

sites. This variability, which contributed to the lack of significance in the statistical tests, probably arose in part from inconsistencies in stimulus delivery. Because it was impossible to apply filter paper directly to the edge of the tongue (when it was attempted, retracting the tongue into the mouth usually caused the paper to move or fall off), the paper had to be placed on the dorsal aspect of the edge. Placement on this area is problematic because the edge of the tongue is indistinct, and varies in shape from one individual to the next. Consequently, the extent to which ethanol actually reached the side of the tongue undoubtedly varied from trial to trial and from subject to subject.

The discontinuities in the psychophysical functions for perceived irritation observed in Experiment 1 (for a concentration of $65 \%$ ) may have occurred again (although less convincingly) in the three functions in Figure 4. The data from the most sensitive site near the edge of the tongue correspond well to the upper four data points from the tonguetip (see Figure 2). The same shape is reflected in the data from the midline of the tongue, which is not entirely consistent with the function obtained on the midline in Experiment 1. However, the use in the present experiment of only four concentrations of ethanol renders conclusions about the forms of the functions tenuous; the functions are less well defined and the context within which subjects judged perceived irritation was different than in Experiment 1 (i.e., the range of intensities was smaller).

\section{EXPERIMENT 3}

After differences were observed in ethanol irritation at different lingual sites, the question arose as to the factors responsible for those differences. The two most likely causes were differences in either the density or the depth of trigeminal nerve endings at the different locations on the tongue. Histological data support both possibilities. Dixon (1962) reported that free nerve endings were both more numerous and more superficially located toward the 
anterior portion of the tongue; Farbman and Hellekant (1978) found that fungiform papillae, which are most prevalent on the anterior of the tongue, were heavily innervated with trigeminal afferents; and, as was noted above, Duner-Engstrom et al. (1986) surveyed the lingual tissue for substance-P immunoreactive fibers and found them only in the epithelium of the anterior region of the tongue (in humans). The latter result was particularly important because substance $P$ has been associated with unmyelinated nerve endings that are likely to subserve chemical irritation (e.g., Adriaensen, Gybels, Handwerker, \& Van Hees, 1980).

Because in Experiments 1 and 2 the response latency to ethanol irritation was positively correlated with perceived intensity of irritation, it seemed reasonable that the depth of the trigeminal nerve endings was likely to be a factor in the perception of irritation. Ethanol should reach nerve endings lying shallow in the epidermis sooner and at a higher concentration than it should reach those lying deeper in the epidermis. However, the number of nerve endings stimulated could also be a significant factor if spatial summation occurs in the sensory system(s) that mediate the burning sensation. Because spatial summation involves the integration of activity from different afferents, a more densely innervated area would (for a given stimulus) yield a more intense and perhaps more rapidly developing sensation of irritation than would a more sparsely innervated area. Experiment 3 was therefore designed to measure the extent of spatial summation of chemical irritation on the tongue.

\section{Method}

Subjects. Ten young adults (6 females and 4 males; average age, 24 years) were paid to participate. A screening procedure eliminated potential subjects who had distinct creases or valleys along the midline of the tongue. This was done to increase the likelihood that the filter paper containing the ethanol stimulus would stay in full contact with the surface of the tongue when the paper was placed across the midline. About half of the potential subjects screened were eliminated because their tongues were insufficiently flat.

Apparatus and Procedure. The stimuli were three sizes of filter paper (Whatman No. 1) saturated with four different concentrations of ethanol: $55 \%, 65 \%, 75 \%$, and $85 \%$ (by volume). The paper rectangles measured $0.5 \times 1.0 \mathrm{~cm}\left(0.5 \mathrm{~cm}^{2}\right), 1.0 \times 1.0 \mathrm{~cm}\left(1.0 \mathrm{~cm}^{2}\right)$, and $2.0 \times 1.0 \mathrm{~cm}\left(2 \mathrm{~cm}^{2}\right)$. When presented to the tongue, the pieces were placed $3 \mathrm{~cm}$ proximal to the tonguetip and were oriented so that their $1-\mathrm{cm}$ width was aligned with the longitudinal (rostrocaudal) plane of the tongue. This meant that increasing stimulus size was equivalent to lengthening the stimulus in the transverse plane (i.e., across the tongue). Area was increased in this manner because Experiment 2 had shown that the sensitivity of the tongue was more uniform in the transverse plane than in the longitudinal plane. Only a small change in response time and no (statistically significant) change in perceived intensity was observed when a stimulus was presented halfway between the edge and the midline of the tongue (Figures 3 and 4). It was therefore considered reasonable to attribute any changes in sensitivity associated with increasing stimulus size to the recruitment of more afferents, rather than to the spread of stimulation to more sensitive areas.

The procedures for applying and removing the stimulus and for obtaining response times and magnitude estimates of perceived irritation were the same as in Experiment 1. A 1-min intertrial in- terval was again imposed, during which the subjects rinsed with $38^{\circ} \mathrm{C}$ deionized water.

\section{Results}

Figure 5 shows the median response times (medians were again used because at the weakest concentration some subjects failed to feel irritation within the 60 -sec observation period); Figure 6 shows the arithmetic means of the normalized magnitude estimates. The effect of stimulus size on latency to onset of irritation was significant overall [Friedman $\chi^{2}(2)=28.6, p<.002$ ], despite an inversion in the response times for the two smaller areas at a concentration of $65 \%$ ethanol. Because of this inversion, it is difficult to arrive at an estimate of the effect on response time of doubling stimulus size from 0.5 to $1.0 \mathrm{~cm}^{2}$. However, a comparison of the data from the $0.5-$ and $2.0-\mathrm{cm}^{2}$ conditions indicates that a fourfold increase in size produced an average increase in response latency of about $7.0 \mathrm{sec}$.

Increasing the size of the stimulus also had a statistically significant effect on the perceived intensity of irritation [univariate repeated measures ANOVA, $F(2,22)=$ $8.89, p=.01$; the size $\times$ concentration interaction was not significant], but the effect was primarily attributable to the differences between the largest and smallest stimuli. Although both measures of sensitivity provide evidence that the sensory system that mediates ethanol irritation integrates neural activity spatially, the data also demonstrate that integration is partial rather than complete. The data for perceived intensity of irritation also raise the possibility that summation may be greater when stimulus size is larger. As can be seen in Figure 6, the largest stimulus consistently produced a stronger irritation than did the intermediate stimulus, whereas the intermediate stimulus produced irritations very similar to those produced by the smallest stimulus. As is the case with the response latency data, the overlap of the mean magnitude estimates for the two smaller areas makes it impossible to arrive at a single number that describes the degree of spatial sum-

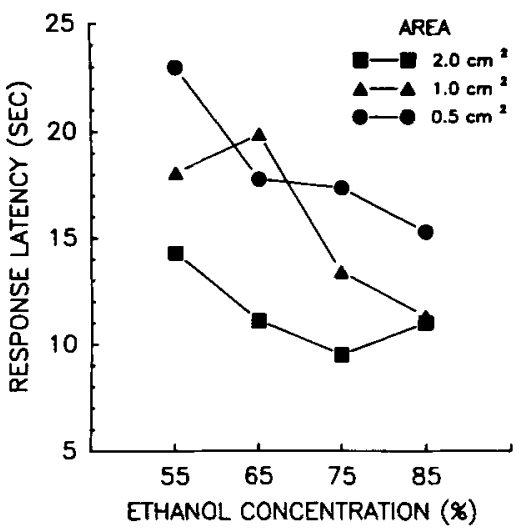

Figure 5. Median latencies for detection of irritation as a function of concentration, shown for three stimulus sizes. The stimuli were applied across the midline of the tongue $3 \mathrm{~cm}$ posterior to the tip. 


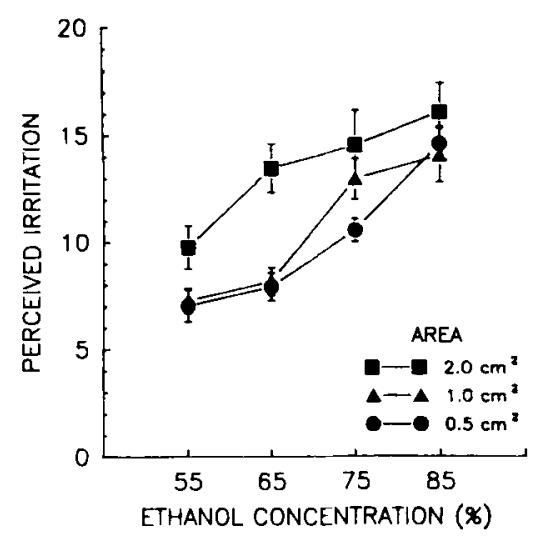

Figure 6. Mean perceived intensity as a function of ethanol concentration for three stimulus sizes. Vertical bars indicate standard errors of the means.

mation. The minimal amount of summation between the two smaller areas means either that for oral irritation magnitude estimation is too coarse a psychological measure to detect small amounts of summation, or that stimulus size must exceed some minimum dimension before spatial summation begins to contribute significantly to perceived irritation.

\section{EXPERIMENT 4}

Experiment 3 showed that a modest amount of summation could be measured when the total area of stimulation was increased by enlarging the stimulus at a single site. Another way to assess spatial summation is to increase the total area of stimulation by adding a second stimulus at another site. Given that lingual sensitivity is spatially heterogeneous, the latter kind of analysis may afford a better measure of spatial summation. That is, if the second stimulus is placed on a corresponding contralateral location, the possibility of obtaining spurious summation due to larger stimuli spreading to more sensitive areas can be avoided. The addition of a contralateral site also tests whether summation can occur across the midline, and hence whether summation can be considered a central phenomenon (i.e., one not restricted to the receptive field of a single afferent).

Spatial summation was therefore explored in Experiment 4 by asking subjects to rate perceived irritation when either one or two ethanol stimuli were applied to the tongue. Response latency was no longer recorded, because even though the stimulator that was used was designed for simultaneous presentation of two stimuli, it could not be guaranteed that the two pieces of filter paper would touch the tongue at precisely the same time.

\section{Method}

Subjects. Twelve young adults (7 females and 5 males; average age, 24 years) were paid to participate. Most were members of the technical staff at the Monell Chemical Senses Center who had participated in other psychophysical studies.
Apparatus and Procedure. Ethanol was delivered to the tongue via saturated filter-paper disks of the same size as those used in Experiment $1\left(0.38 \mathrm{~cm}^{2}\right)$. Five concentrations of ethanol $(45 \%$, $55 \%, 65 \%, 75 \%$, and $85 \%$, by volume, in deionized water) were tested. So that two filter-paper disks could be placed on the tongue at the same time and at a fixed distance from one another, two forceps were mechanically joined so that their jaws opened simultaneously when they were pinched between the thumb and forefinger of one hand. This was accomplished by gluing two rectangular plastic blocks (each $9 \times 13 \times 30 \mathrm{~mm}$ ) onto opposite sides of the forceps' handles. The plastic blocks rigidly joined the forceps, fixing the space between their tips at $2 \mathrm{~cm}$. The resulting "stereo" forceps were operated by pinching together the opposing pieces of plastic.

In addition to the center-to-center spacing of $2 \mathrm{~cm}$ (which meant that the center of each stimulus was located $1 \mathrm{~cm}$ from the midline), the stimuli were also placed $1 \mathrm{~cm}$ proximal to the tip of the tongue. The food-color marker was replaced in this experiment by a line etched across the handles of the forceps $1 \mathrm{~cm}$ from their tip. The experimenter aligned this mark with the tip of the tongue before opening the forceps and placing the filter papers on the tongue.

On each trial the subjects received two filter-paper disks: either both were saturated with equal concentrations of ethanol or one was saturated with an ethanol solution and the other with deionized water. Every stimulus pair was presented three times per session and the order of presentation was randomized by computer. As in the preceding experiments, the subject pressed a key when he/she felt the disks touch the tongue. The keypress started a computer clock, and a tone sounded $9.5 \mathrm{sec}$ later. At that signal the subject protruded the tongue to allow the experimenter to remove the disks. The subject then responded verbally with a magnitude estimate of the overall sensation of irritation he/she had felt on the tongue. The subjects rinsed vigorously between trials with deionized water heated to approximately $38^{\circ} \mathrm{C}$. The intertrial interval was $60 \mathrm{sec}$.

\section{Results}

Figure 7 depicts the normalized magnitude estimates for the two conditions of the experiment: bilateral (both sides of the tongue received ethanol) and unilateral (one side of the tongue received ethanol and the other side received deionized water). The data are arithmetic means of normalized magnitude estimates.

The results indicate that sensations of irritation were stronger when two ethanol stimuli were presented than

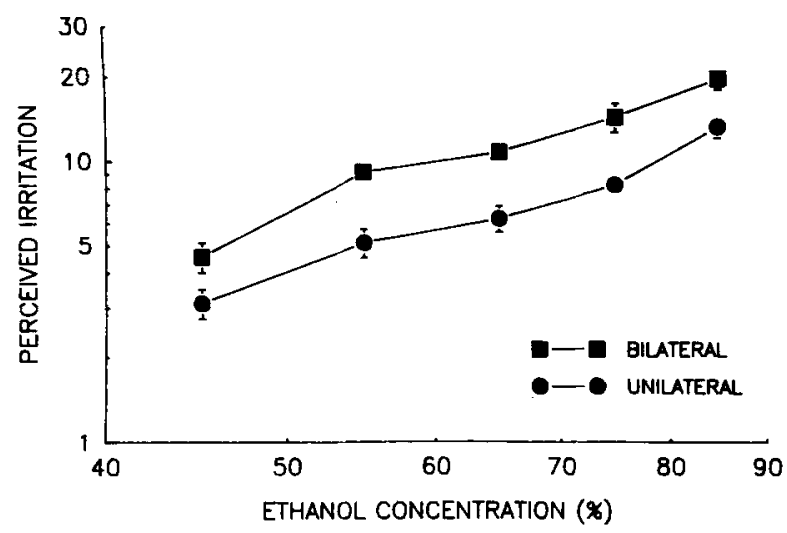

Figure 7. Mean perceived irritation as a function of ethanol concentration when both sides of the tongue received ethand (bilateral) and when one side received ethanol and the other side received deionized water. The stimuli were presented on corresponding bilateral sites. Vertical bars indicate standard errors of the means. 
when one ethanol stimulus was presented. The data have been plotted on $\log$-log coordinates to illustrate that the amount of summation is roughly proportional throughout the intensity range. A univariate repeated measures ANOVA confirmed that the effect of stimulus number was significant $[F(1,11)=47.144, p<.001]$, whereas the interaction between stimulus number and concentration was not. The data from both conditions conform reasonably well to power functions ( $r s \geq .98$ ) and have virtually identical slope constants ( 2.10 for the unilateral condition and 2.11 for the bilateral condition).

The most direct way to assess the degree of summation these data represent is to compare the concentrations of ethanol that would be required to produce equal levels of perceived irritation when either one or two stimuli were applied. Calculations based upon the best-fitting power functions indicate that equal levels of irritation would be obtained if ethanol concentration were reduced by approximately $21 \%$ when a second site was added. Because a decrease in concentration of $50 \%$ following the addition of a second stimulus would represent $100 \%$ (complete) summation, the $21 \%$ value represents $42 \%$ summation. Therefore, like spatial summation at a single site, summation between two (bilateral) sites is significant but partial.

\section{EXPERIMENT 5}

Experiment 4 showed that summation occurs across the midline when ethanol concentration is equal on the two sites; Experiment 5 was designed to explore what happens when concentrations differ at the two sites. Data from bilateral experiments on nasal trigeminal sensitivity (Garcia-Medina \& Cain, 1982) indicate that when a high concentration is paired with a low concentration, the higher concentration dominates the perception (seemingly inhibiting the weaker input) and summation is reduced. Therefore, Experiment 5 included tests of four different ethanol concentrations presented in all possible combinations to one or both sides of the tongue.

\section{Method}

Subjects. Twenty-five young adults ( 12 males and 13 females; average age, 25.2 years) were paid to participate in the experiment; a few subjects had served in one or more of the preceding experiments, but did not know their outcomes.

Apparatus and Procedure. The ethanol concentrations tested were $0 \%, 40 \%, 55 \%, 70 \%$, and $85 \%$. Presentation of the stimuli to the tongue was the same as in Experiment 4, except that the filterpaper disks were left on the tongue for $15 \mathrm{sec}$ rather than $10 \mathrm{sec}$. The longer period of stimulation was used to help ensure that sensations of irritation would have time to develop at the lowest ethanol concentration (40\%). In Experiment 4 the $45 \%$ solution was sometimes barely perceptible, and Experiments 1 and 2 had revealed that latency to sensation onset was inversely related to concentration.

Each combination of concentrations (which included pairings with the deionized water blank on one side of the tongue) was presented twice in random sequence to each subject in a single 1-h session. Perceived irritation was again assessed using the method of magnitude estimation, and the subjects were told to base their estimates on the peak sensation of irritation perceived during the $15-\mathrm{sec}$ ob- servation interval. As in the preceding experiment, emphasis was placed upon judging the overall, bilateral sensation of irritation. Subjects rinsed with $38^{\circ} \mathrm{C}$ deionized water during the 60 -sec intertrial interval.

\section{Results}

The normalized mean magnitude estimates are shown in Figure 8. The data indicate that spatial summation occurred whether the bilateral stimuli had the same or different concentrations. A univariate repeated measures ANOVA confirmed the significance of the spatial effects $[F(4,96)=40.882, p<.001]$ and indicated that the interaction term was not significant $[F(12,288)=1.19$, $p=.29$ ]. The absence of an interaction suggests that on average the increase in perceived intensity produced by the addition of a second stimulus to the contralateral side of the tongue was approximately the same, regardless of the concentration of the stimulus on the ipsilateral side. The data do, however, converge visibly at higher concentrations (also noticeable on linear coordinates), which raises the possibility that there is at least a tendency for summation to be less when weaker stimuli are added to stronger stimuli. This trend, although not sufficient to produce a significant interaction, is worth mentioning because it is consistent with what was reported for bilateral nasal irritation (Garcia-Medina \& Cain, 1982). GarciaMedina and Cain suggested that an inhibitory as well as a summating process occurs between the nostrils. If inhibition takes place across the tongue, it is apparently weaker than the inhibition that seems to take place between nostrils.

However, firm conclusions about the strength of bilateral interactions should not be drawn from the present data. Despite the relatively large number of subjects tested in the experiment (25), the data remain imprecise. Much of the variability in the data probably resulted from the inverse relationship that exists between ethanol concentration and the latency to sensation onset. Thus, when

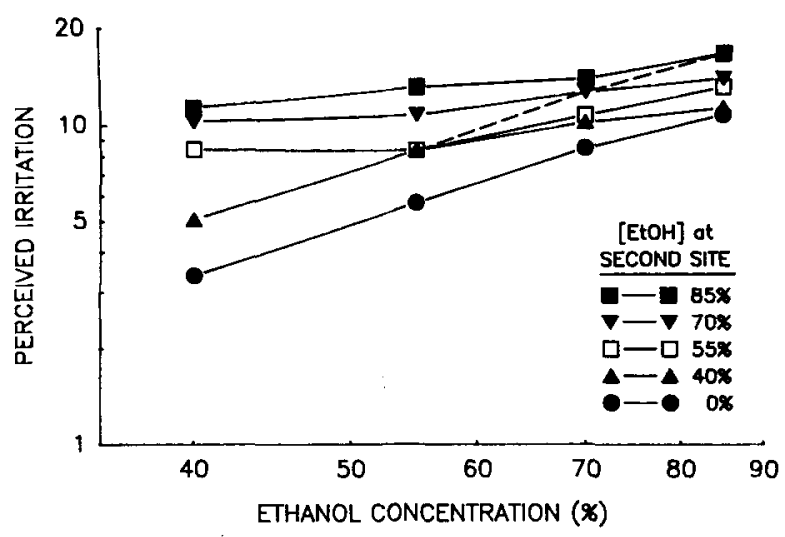

Figure 8. Mean perceived irritation as a function of ethanol concentration for pairs of bilateral stimuli having the same or different concentrations $(0 \%, 40 \%, 55 \%, 70 \%$, and $85 \%)$. The dashed line connects the points generated when equal concentrations were presented to both sides of the tongue (as in Experiment 4; see Figure 7). 
stimulus concentrations differed at the two sites, so too did the latencies of the resulting sensations. The subjects were forced to judge overall intensity when sensations on opposite sides of the tongue were appearing and reaching peak values at different times. This unavoidable circumstance made it difficult for the subjects to respond consistently. Over all the conditions of the experiment, the standard errors of the means (SEMs) ranged between approximately $4.5 \%$ and $10.5 \%$ of the mean magnitude estimates, and tended to be greatest when ethanol concentrations differed across the tongue. In the most extreme cases, when $85 \%$ and $70 \%$ solutions were paired with $40 \%$ solutions, the SEMs increased by $77.6 \%$ and $91.3 \%$ over what they were when the high concentrations were paired with themselves. (SEMs were omitted from Figure 8 because the frequent overlap of the data points rendered their depiction ambiguous.)

When equal concentrations were applied to both sides of the tongue (triggering simultaneous rather than sequential sensations), the results were in close agreement with those of Experiment 4. Calculations based upon bestfitting power functions $(r \mathrm{~s} \geq .997)$ indicated that the concentration required to produce a given level of irritation was $22.5 \%$ lower when two stimuli were applied than when one stimulus (plus deionized water) was applied. The estimate for the same circumstances in Experiment 4 was $21 \%$.

\section{GENERAL DISCUSSION}

This study demonstrates two basic facts about the tongue's sensitivity to ethanol. First, sensitivity is spatially heterogeneous. When measured in terms of response latency, sensitivity varies significantly both along and across the tongue; when measured in terms of perceived intensity, sensitivity varies significantly in the longitudinal plane while exhibiting a nonsignificant trend toward higher intensities in more lateral regions. Second, response latency and perceived intensity vary with the amount of lingual skin that is stimulated, indicating that spatial integration takes place.

Experiments 1 and 2 showed that the spatial pattern of sensitivity to chemical irritants is similar to the spatial pattern of sensitivity to gustatory and other cutaneous stimuli. This is not surprising in view of the dense innervation of fungiform papillae by the trigeminal nerve (Farbman \& Hellekant, 1978) and the generally greater density and superficiality of unmyelinated nerve endings near the tip of the tongue (Dixon, 1962). The anterior of the tongue (and, to a lesser extent, the side of the tongue) is therefore an extraordinarily sensitive, multimodal sensory area.

The first two experiments also indicated that the irritating qualities of a chemical stimulus should become perceptible only after its gustatory qualities have appeared. Studies of gustatory reaction time have generally revealed response latencies of $1 \mathrm{sec}$ or less for moderately intense stimuli (e.g., Yamamoto et al., 1982; Yamamoto \&
Kawamura, 1981). In contrast, not even at the tip of the tongue was the highest concentration of ethanol- $85 \%$. or 170 proof-perceived in less than about $2 \mathrm{sec}$. Although it is quite possible that other areas of the oral cavity give rise to sensations of irritation more rapidly than the tongue, it is likely that stimuli that provoke both a taste and an irritation will be perceived as having a taste before they are perceived as having an irritation. A similar situation occurs in the nose, where the response latency to odors is briefer than the response latency to volatile irritants (Cain, 1976).

Although it is possible that the long latencies for irritation are due to the depth of trigeminal end organs in the epithelium, it is also possible that temporal summation plays a role. It has been shown by Adriaensen et al. (1980) that after application of an irritant, activity can occur in first-order afferents for $10 \mathrm{sec}$ or longer before sensations of irritation are experienced. These results, obtained in awake humans, stand as intriguing evidence that the relatively long latency to the onset of irritation derives at least in part from integrative mechanisms in the central nervous system.

Experiment 3 demonstrated that response latency varies inversely with stimulus size, and Experiments 4 and 5 showed that perceived intensity varies directly with stimulus size. Both results indicate that some degree of spatial summation occurs in the sensory systems that are stimulated by ethanol. The occurrence of summation indicates that innervation density probably contributes to regional differences in sensitivity. Thus, although other factors (such as the depth of the nerve endings in the mucosal epithelium) may play major roles in establishing local sensitivity, the number of nerve fibers that the irritant reaches is also important.

The finding that summation is less than complete seems consistent with the notion that ethanol's irritating effects are mediated by at least some of the same nerve fibers that mediate heat pain. Psychophysical studies in humans had previously found relatively small amounts of summation when stimulus temperature rose toward noxious levels (Greene \& Hardy, 1958; Stevens \& Marks, 1971). Although a recent study by Kojo and Pertovaara (1987) challenged this notion by showing spatial summation of heat pain comparable to spatial summation of warmth, it is not clear what criteria the subjects used in that study to identify the heat pain threshold. There are, in any case, data from electrophysiological experiments that indicate that the afferents activated by other chemical irritants are c-polymodal nociceptors and A-delta nociceptors (Adriaensen, Gybels, Handwerker, \& Van Hees, 1983; Foster \& Ramage, 1981; Kenins, 1982; Szolcsányi, 1987; Van Hees \& Gybels, 1981), and percutaneous neural recordings in humans (mentioned above) have provided indirect evidence that spatial and/or temporal summation is a characteristic of the sensory systems associated with these afferents (Adriaensen et al., 1980). The present finding of partial summation is therefore consistent with the 
bulk of previous evidence that points to limited summation in the nociceptive sense(s). It should be noted, however, that the only study that attempted to determine which types of nerve fibers were activated by ethanol applied to the tongue (in rats) produced no evidence of nociceptor involvement (Hellekant, 1967).

Viewed overall, the data of all five of the present experiments provide the following basic picture of the spatiotemporal aspects of ethanol irritation during ingestion: When an alcoholic beverage bathes the tongue, sensations of irritation appear first at the tip and then spread to less sensitive areas at the sides, middle, and back of the tongue (providing ethanol concentration is sufficiently high to produce sensations in the less sensitive regions). As ethanol reaches more and more receptors, neural activity is integrated and sensation intensity grows. The same sequence of events undoubtedly occurs in other oral structures as the full impression of the ethanol "burn" develops. Simple introspection seems to verify this sequence of events and implies further that when solutions are swallowed rather than expectorated, two areas of the mouth not tested in this study-the soft palate and the oralpharyngeal region-are at least as important as the tongue for sensing the irritation ethanol produces.

The present results point to a need for future studies of the temporal profiles of trigeminal, gustatory, and olfactory sensations during the development of complex flavors. It seems likely, for example, that the practice of sipping and savoring wines and spirits owes largely to the desirability of allowing adequate time for all three sensory components to appear and combine into a unified sensation of flavor.

\section{REFERENCES}

adriaensen, H., Gybels, J., Handwerker, H. O., \& Van Hees, J. (1980). Latencies of chemically evoked discharges in human cutaneous nociceptors and of the concurrent subjective sensations. Neuroscience Letters, 20, 55-59.

Adruannsen, H., Gybels, J., Handwerker, H. O., \& Van Hees, J. (1983). Response properties of thin myelinated (A-delta) fibers in human skin nerves. Journal of Neurophysiology, 49, 111-122.

CAIN, W. S. (1976). Olfaction and the common chemical sense: Some psychophysical contrasts. Sensory Processes, 1, 57-67.

Dixon, A. D. (1962). The position, incidence and origin of sensory nerve terminations in the oral mucous membrance. Archives of Oral Biology, 1, 39-48.

Duner-Engstrom, M., Fredholm, B. B., LARSSON, O., LundBerg, J. M., \&ARIA, A. (1986). Autonomic mechanisms underlying capsaicin-induced oral sensations and salivation in man. Journal of Physiology, 373, 87-96.

Farbman, A. I. Hellekant, G. (1978). Quantitative analyses of the fiber population in rat chorda tympani nerves and fungiform papillae. American Joumal of Anatomy, 153, 509-521.

Foster, R. W., RAMAGE, A. G. (1981). The action of some chemical irritants on somatosensory receptors of the cat. Neuropharmacology, 20, 191-198.

Garcia-Medina, M. R., Cain, W. S. (1982). Bilateral integration in the common chemical sense. Physiology \& Behavior, 29, 349-353.

GREEN, B. G. (1984). Thermal perception on lingual and labial skin. Perception \& Psychophysics, 36, 209-220.

GreEN, B. G. (1985). Heat pain thresholds in the oral-facial region. Perception \& Psychophysics, 38, 110-114.

GREEN, B. G. (1986). Sensory interactions between capsaicin and temperature in the oral cavity. Chemical Senses, 11, 371-382.

GREENE, L. C., \& HARDY, J. D. (1958). Spatial summation of pain. Jourmal of Applied Physiology, 13, 457-464.

HeLLEKANT, G. (1967). Action and interaction of ethyl alcohol and some other substances on the receptors of the tongue. In T. Hayashi (Ed.), Olfaction and Taste II. New York: Pergamon Press, pp. 465-479.

Kenins, P. (1982). Responses of single nerve fibers to capsaicin applied to the skin. Neuroscience Letters, 29, 83-88.

Kojo, I., \& Pertovanra, A. (1987). The effects of stimulus area and adaptation temperature on warm and heat pain thresholds in man. International Joumal of Neuroscience, 32, 875-880.

Konietzny, F., Hensel, H. (1983). The effect of capsaicin on the response characteristics of human c-polymodal nociceptors. Journal of Thermal Biology, 8, 213-215.

LAWLESS, H. (1984). Oral chemical irritation: Psychophysical properties. Chemical Senses, 9, 143-155.

MARTIN, S., \& PANGBoRn, R. M. (1970). A note on the responses to ethyl alcohol before and after smoking. Perception \& Psychophysics, 8, 169-170.

Parker, G. H., Stabler, E. M. (1913). On certain distinctions between taste and smell. American Journal of Physiology, 32, 230-240.

Rozin, P., EberT, L., Schull, J. (1982). Some like it hot: A temporal analysis of hedonic responses to chili pepper. Appetite, 3, 13-22.

SmITH, S. E. (1972). Taste thresholds in drug addicts and alcoholics. British Journal of Addiction, 67, 317-321.

Stevens, D. A., \& Lawless, H. T. (1986). Putting out the fire: Effects of tastants on oral chemical irritation. Perception \& Psychophysics, 39, 346-350.

Stevens, J. C., \& Marks, L. E. (1971). Spatial summation and the dynamics of the warmth sense. Perception \& Psychophysics, 9, 391-398.

Szolcsínyi, J. (1987). Capsaicin and nociception. Acta Physiologica Hungarica, 69, 323-332.

VAN HeEs, J., \& GybeLs, J. (1981). C nociceptor activity in human nerve during painful and non-painful stimulation. Journal of Neurology, Neurosurgery \& Psychiatry, 44, 600-607.

Wilson, C. W. M., OBRien, C., MacAirt, J. G. (1973). The effect of metronidazole on the human taste threshold to alcohol. British Journal of Addiction, 68, 99-110.

Yamamoto, T., Kato, T., Matsuo, R., Araie, N., Azuma, S., \& KAWAMURA, Y. (1982). Gustatory reaction time under variable stimulus parameters in human adults. Physiology \& Behavior, 29, 79-84.

YAmAMOTO, T., \& KAwAMURA, Y. (1981). Gustatory reaction time in human adults. Physiology \& Behavior, 26, 715-719.

(Manuscript received November 16, 1987; revision accepted for publication February 16, 1988.) 